\title{
Microstructures and the corresponding magnetic properties of half-doped $\mathrm{Nd}_{0.5} \mathrm{Sr}_{0.5} \mathrm{MnO}_{3}$ films
}

\author{
V. G. Prokhorov, ${ }^{1}$ Y. H. Hyun, ${ }^{2}$ J. S. Park, ${ }^{2}$ J. B. Kim, ${ }^{2}$ G. H. Kim, ${ }^{2}$ Y. S. Lee, ${ }^{2}$ \\ Y. P. Lee, ${ }^{2, a)}$ and V. L. Svetchnikov ${ }^{3}$ \\ ${ }_{1}^{1}$ Institute for Metal Physics, NASU, Kiev 03142, Ukraine \\ ${ }_{2}^{2}$-Psi and Department of Physics, Hanyang University, Seoul 133-791, Republic of Korea \\ ${ }^{3}$ National Center for HREM, TU Delft, 2628AL Delft, The Netherlands
}

(Received 22 June 2008; accepted 25 September 2008; published online 17 November 2008)

\begin{abstract}
The microstructural and magnetic properties of amorphous, nanoclustered, and self-organized bilayer $\mathrm{Nd}_{0.5} \mathrm{Sr}_{0.5} \mathrm{MnO}_{3}$ films, prepared by rf magnetron sputtering, are investigated. The amorphous film was found to be a typical paramagnet with free motion of the individual Mn spins, and the magnetic properties are well described on the basis of the Curie-Weiss approximation. The nanoclustered film manifests magnetic properties mimicking those of superparamagnetic particles following nonclassical magnetic dynamics. The self-organized bilayer film demonstrates a negative exchange bias typical of a ferromagnet/antiferromagnet hybrid system, in spite of the fact that both layers have a ferromagnetic origin. The magnetic properties of the films are discussed in detail on the basis of modern theoretical models. (C) 2008 American Institute of Physics.
\end{abstract}

[DOI: $10.1063 / 1.3020529]$

\section{INTRODUCTION}

Hole-doped manganites $L_{1-x} A_{x} \mathrm{MnO}_{3}$, where $L$ and $A$ are a trivalent lanthanide ion and a divalent alkaline-earth ion, respectively, have attracted considerable attention due to their interesting fundamental science and potential for applications. ${ }^{1}$ Their peculiar physical behavior originates from the strong coupling between the magnetic, structural, electronic, and orbital degrees of freedom and is manifested more greatly in the half-doped perovskite compounds. $\mathrm{Nd}_{1-x} \mathrm{Sr}_{x} \mathrm{MnO}_{3}$ is a typical system whose ground state varies from a ferromagnetic (FM) metal to an $A$-type antiferromagnetic (AFM) metal (or insulator at $x \geq 0.6$ ) when the Sr doping crosses $0.5^{2}$ In addition, a charge-ordered (CO) phase with the AFM charge exchange (CE)-type structure is formed in a narrow doping range of $0.48 \leq x \leq 0.51{ }^{3}$ The band structure calculations reveal that the energy difference between FM and AFM phases is very small at around $0.01 \mathrm{eV} /$ atom. As a consequence, the ground state of $\mathrm{Nd}_{0.5} \mathrm{Sr}_{0.5} \mathrm{MnO}_{3}$ (NSMO) is highly dependent on the degree of crystallinity, the lattice strain, the chemical homogeneity, and the clustering of the microstructure, which are controlled by the fabrication technique. Thus the $\mathrm{CO}$ state, for example, is observed in the NSMO film with a tensile in-plane strain, ${ }^{5}$ while it is not found in the film with a compressive one. ${ }^{6}$ The CO AFM CE-type state can be suppressed by increased film thickness, ${ }^{7}$ by high hydrostatic pressure, ${ }^{8,9}$ decreased grain size in polycrystalline samples, ${ }^{10,11}$ or applied electric field. ${ }^{12}$ On the other hand, the influence of quenched structural disorder and fine-crystalline clustering on the magnetic and the charge ordering in this compound is still poorly understood.

In this paper we report the experimental results for NSMO films with different microstructures. The observed

${ }^{a)}$ Electronic mail: yplee@ hanyang.ac.kr. evidences for the superparamagnetic (SPM) state and the negative exchange bias (EB) effect are discussed in detail.

\section{EXPERIMENTAL TECHNIQUES}

The NSMO films were prepared by the rf magnetron sputtering using a so-called soft (or powder) target. ${ }^{13}$ The substrate was a $\mathrm{LaAlO}_{3}$ (LAO) (100) single crystal with an out-of-plane lattice parameter $c \simeq 0.379 \mathrm{~nm}$ for the pseudocubic symmetry. The substrate temperature $\left(T_{\text {sub }}\right)$ during deposition was varied from 20 to $750{ }^{\circ} \mathrm{C}$, and only the as-deposited films were used for study. The thickness of all the films was $\simeq 100 \mathrm{~nm}$. The $\theta-2 \theta$ x-ray diffraction (XRD) patterns were obtained using a Rigaku diffractometer with $\mathrm{Cu} K \alpha$ radiation. The lattice parameters, evaluated directly from the XRD data, were plotted against $\cos ^{2} \theta / \sin \theta$. From the intercept of the extrapolated straight line to $\cos ^{2} \theta / \sin \theta=0$, more precise lattice parameters were obtained. The high-resolution electron microscopy (HREM) and electron-diffraction (ED) studies were carried out using a Philips CM300UT-FEG microscope with a field emission gun operated at $300 \mathrm{kV}$. The point resolution of the microscope was in the order of $0.12 \mathrm{~nm}$. The cross-sectional specimens were prepared by the standard techniques using mechanical polishing followed by ion-beam milling at a grazing incidence. The magnetic measurements were performed by using a Quantum Design superconducting quantum interference device magnetometer.

\section{MICROSTRUCTURE}

Figure 1 presents the $\theta-2 \theta$ XRD scans for the films deposited at $T_{\text {sub }} \simeq 20{ }^{\circ} \mathrm{C}$ (a), $300{ }^{\circ} \mathrm{C}$ (b), and $750{ }^{\circ} \mathrm{C}$ (c). The film deposited at the lowest substrate temperature manifests only the very fuzzy Bragg peaks near the $(00 l)$-like reflections from LAO substrate, which is typical for the fully disordered (or amorphous) crystal structure. ${ }^{14}$ The inset in Fig. 


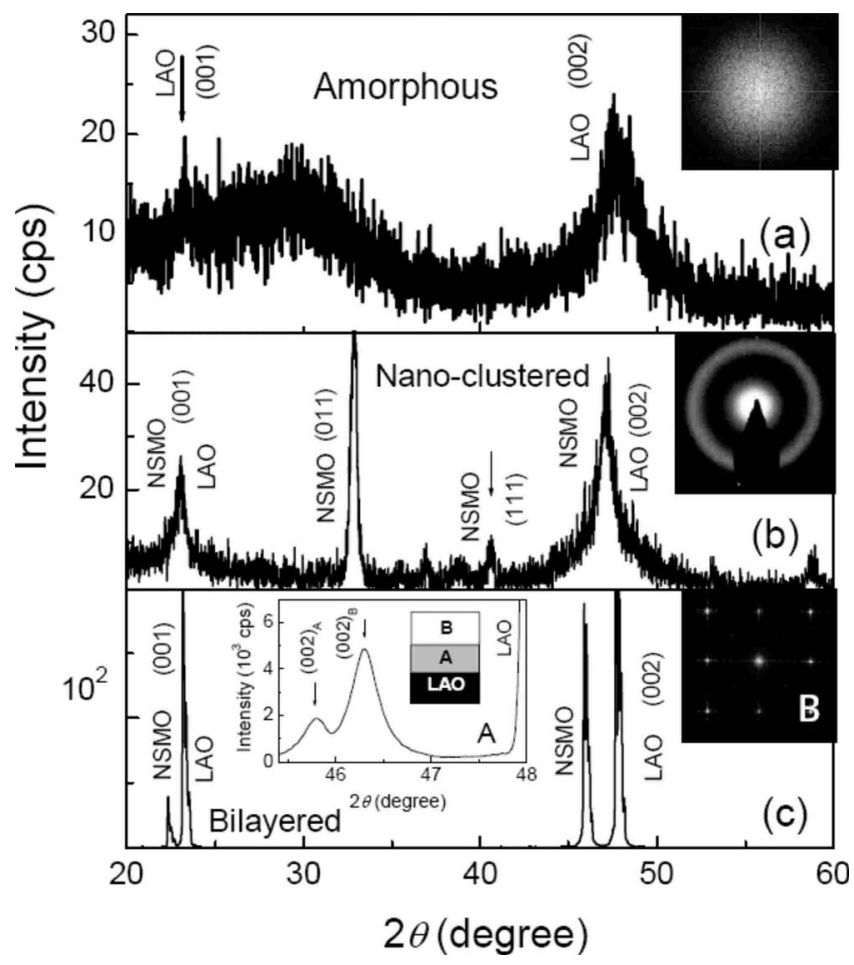

FIG. 1. $\theta-2 \theta$ XRD scans for the NSMO films, deposited at (a) $20^{\circ} \mathrm{C}$, (b) $300{ }^{\circ} \mathrm{C}$, and (c) $750{ }^{\circ} \mathrm{C}$. The inset in (a) and inset (B) in (c) are the FFTs of corresponding HREM images. The inset in (b) is the corresponding [010] zone-axis ED pattern taken at RT. Inset (A) in (c) is the XRD scan of the (002) Bragg peak for the film deposited at $750{ }^{\circ} \mathrm{C}$. Arrows indicate the presence of two layers with different crystal structures. NSMO and LAO denote the film and the substrate, respectively.

1(a) displays the fast Fourier transform (FFT) of the crosssectional HREM image (not shown) for this film. It can be seen that the FFT produces a bright uniform halo instead of the rectangular pattern of circular spots which are formed by the regular crystal lattice. Therefore, we conclude that the film deposited at $T_{\text {sub }} \simeq 20{ }^{\circ} \mathrm{C}$ is mainly an amorphous structure.

The film made at an intermediate $T_{\text {sub }} \simeq 300{ }^{\circ} \mathrm{C}$ manifests the $(0 k l)$ - and $(h k l)$-like Bragg peaks in addition to the broad (00l) XRD reflections. Therefore, the deposition in this case results in a polycrystalline microstructure. The ED pattern, represented by the inset in Fig. 1(b), shows a wide ring, which is typical for the nanoclustered disordered structure. ${ }^{14}$ The estimated average size of the nanoclusters turns out to be about $4 \mathrm{~nm}$. This film will be referred hereinafter as the nanoclustered film.

A more unexpected microstructure was found for the film grown at $T_{\text {sub }} \simeq 750{ }^{\circ} \mathrm{C}$. The XRD scan displays only the $(00 l)$ Bragg peaks of high intensity, indicating that the deposition results in a highly $c$-oriented crystal structure [Fig. 1(c)]. This result is confirmed by the FFT of the crosssectional HREM image, represented by inset (B), which reveals a rectangular pattern of circular spots. At the same time, inset (A) exhibits a slight split of the (002) XRD Bragg peak (indicated by arrows), which can be treated as the presence of two layers with different crystal structures, called A and B. A similar two-layer structure has already been observed for the half-doped NSMO film grown by the pulsed

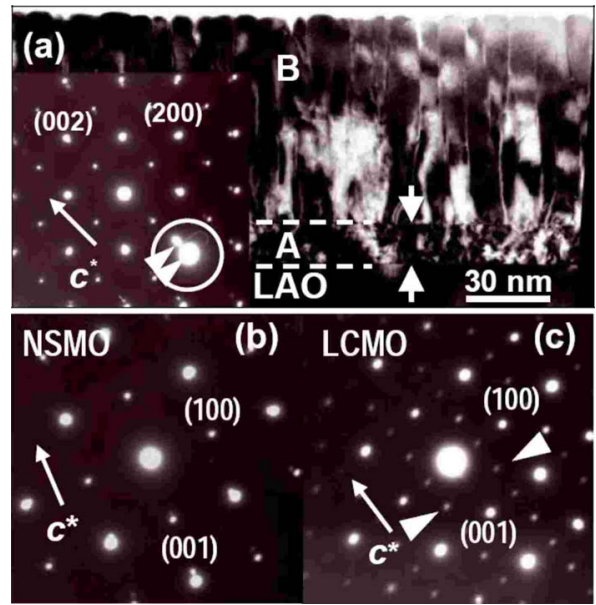

FIG. 2. (Color online) (a) Low-magnification cross-sectional HREM image taken at RT for the self-organized NSMO bilayer film, deposited at $750{ }^{\circ} \mathrm{C}$. $\mathrm{A}$ and $\mathrm{B}$ denote the epitaxial bottom layer and the top nanoclustered columnlike microstructure, respectively. LAO denotes a substrate. The inset [left figure in (a)] is the corresponding [010] zone-axis ED pattern taken at RT. Arrows inside the white ring indicate a slight splitting of the Bragg reflection, which testifies for the presence of two layers with different crystal structures. (b) The same ED pattern taken at $87 \mathrm{~K}$. (c) [010] zone-axis ED pattern taken at $87 \mathrm{~K}$ for the half-doped $\mathrm{La}_{0.5} \mathrm{Ca}_{0.5} \mathrm{MnO}_{3}$ film. The white arrows indicate the superlattice reflections connected with the $\mathrm{CO}$ state.

laser deposition on a LAO at $T_{\text {sub }} \simeq 820^{\circ} \mathrm{C}$. ${ }^{15}$ The bottom (A) and the top (B) layers have an out-of-plane lattice parameters of 0.3896 and $0.3856 \mathrm{~nm}$, respectively. The crosssectional low-magnification HREM image, taken at RT and represented by Fig. 2(a), exhibits the bilayered microstructure more clearly. However, it should be noted that the HREM image prepared at $87 \mathrm{~K}$ remains unchanged. The film includes a well-defined 20-nm-thick bottom epitaxial $A$ layer (indicated by white arrows), while the top $B$ one is in a columnlike nanostructure. The average diameter of a column turns out to be about $10 \mathrm{~nm}$. The ED pattern prepared at RT [see inset in Fig. 2(a)] reveals the formation of a well-known orthorhombic crystal structure in both the top and the bottom layers with the lattice parameters, which are excellently coincident with the XRD analysis and very similar to the bulk: $a \simeq b \simeq 0.385 \mathrm{~nm}$ and $c \simeq 0.389 \mathrm{~nm}$ (A layer) and $0.386 \mathrm{~nm}$ (B layer). 2,9,16 The difference in the out-of-plane parameter is manifested by a slight splitting of the Bragg reflection along the $c^{*}$ direction, which is indicated by arrows inside the white circle in the inset. The bottom A layer has a strained crystal lattice (the $c$ parameter is larger than that of the bulk) due to the epitaxial growth and the large mismatch between the film and substrate lattice parameters. On the other hand, as the thickness of the grown layer exceeds a certain critical value during deposition, the film tends toward stress relaxation by the formation of misfit dislocations or a columnlike microstructure directed normal to the film plane. ${ }^{17}$ This film will be referred to hereinafter as the self-organized bilayer film.

It is significant to note that the ED patterns, prepared at RT [see inset in Fig. 2(a)] and 87 K [Fig. 2(b)], display only fundamental Bragg spots without a trace of the $\mathrm{CO}$ phase, which is observed at $T_{\mathrm{CO}} \simeq 150 \mathrm{~K}$ in the bulk as a rule. The $\mathrm{CO}$ phase is characterized by the appearance of superlattice 


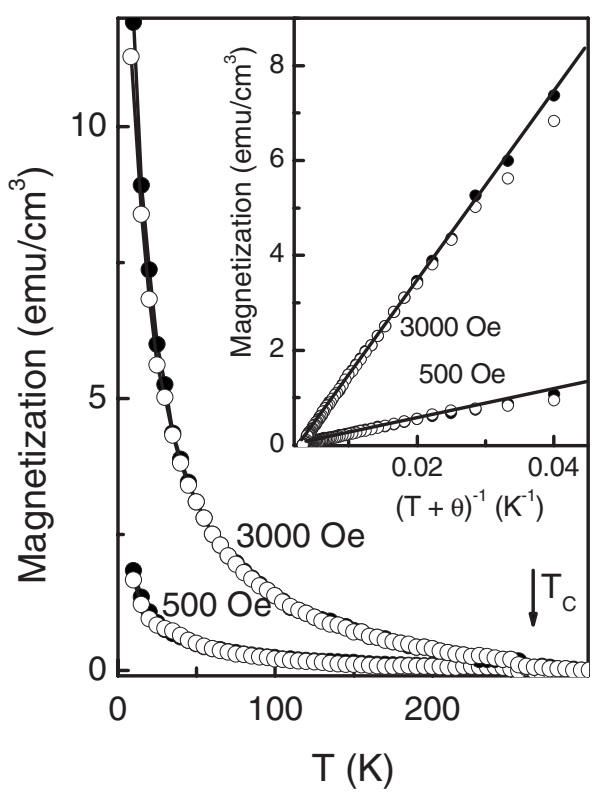

FIG. 3. Temperature dependence of the in-plane FC (solid symbols) and ZFC (open symbols) magnetization for the amorphous NSMO film, measured at different applied magnetic fields. Lines are guides to the eyes. Arrows indicate the location of the Curie point for this compound. The inset is the $M(T)$ vs $1 /(T+\theta)$ plot for the same film.

spots on the ED patterns, with a wave vector $q=a^{*} / 2$, where $a^{*}$ is the reciprocal lattice vector along the $a$ axis for simple pseudocubic symmetry. For comparison, Fig. 2(c) shows the same ED pattern for the half-doped $\mathrm{La}_{0.5} \mathrm{Ca}_{0.5} \mathrm{MnO}_{3}$ film, prepared at the same conditions, in which the $\mathrm{CO}$ state is observed. ${ }^{18}$ The superlattice reflections are indicated by white arrows. No CO phase in the half-doped NSMO film even at $87 \mathrm{~K}$ can be explained as follows. First, the major part of the film (B layer) consists of small-size columnlike clusters that provide the complete suppression of the $\mathrm{CO}$ phase. ${ }^{10,11}$ Second, the bottom A layer has an in-plane compressive strained crystal lattice that also leads to the degradation of the $\mathrm{CO}$ state. $^{6}$

In summary, three kinds of NSMO films with different microstructures were prepared: the nearly amorphous film with the fully disordered crystal structure $\left(T_{\text {sub }} \simeq 20{ }^{\circ} \mathrm{C}\right)$, the nanoclustered polycrystalline film with an average cluster diameter of nearly $4 \mathrm{~nm}\left(T_{\text {sub }} \simeq 300{ }^{\circ} \mathrm{C}\right)$, and the selforganized bilayer film with a homogeneous chemical composition and different microstructures $\left(T_{\text {sub }} \simeq 750{ }^{\circ} \mathrm{C}\right)$. None of the films reveal the trace of $\mathrm{CO}$ state with decreasing temperature even down to $87 \mathrm{~K}$.

\section{EXPERIMENTAL RESULTS}

Figure 3 shows both field-cooled (FC) (solid symbols) and zero-field-cooled (ZFC) (open symbols) temperaturedependent magnetization curves, $M(T)$, for the amorphous film at different magnetic fields. The $M(T)$ behavior is typical for the paramagnetic (PM) materials ${ }^{19}$ and can be written for the entire temperature range as

$$
M^{\mathrm{PM}}(T, H)=\left[\chi_{0}+C_{\mathrm{CW}} /(T+\theta)\right] H,
$$

where $\chi_{0}$ is the temperature-independent susceptibility, and the second term is the Curie-Weiss (CW)-type susceptibility with a constant $C_{\mathrm{CW}}$ and a characteristic temperature $\theta$. The inset shows that the experimental data are well described by the $\mathrm{CW}$ expression with the following fitting parameters: $C_{\mathrm{CW}} \simeq 0.002 \mathrm{emu} \mathrm{K} / \mathrm{T}$ and $\theta=5 \mathrm{~K}$. It is worth noting that the characteristic temperature $\theta$ in this case does not correspond to the AFM or the FM exchange integral within the mean-field theory but manifests only the deviation of the $M(T)$ experimental curve from the Curie-law approximation. An additional temperature term in the Curie law may be necessary in order to account for the presence of the small fraction of diluted nanocrystalline inclusions in the amorphous matrix of film. This is confirmed by a minor kink on the experimental $M(T)$ dependence near the Curie point (see Fig. 3) and a slight splitting between FC and ZFC $M(T)$ curves at low temperatures (see the inset). At the same time, the effective magnetic moment estimated from $C_{\mathrm{CW}}$ was $\mu_{\mathrm{eff}} \simeq 4.4 \mu_{B} / \mathrm{Mn}$, which is nearly coincident with the theoretical value, $\mu_{\text {eff }}^{\text {theor }} \simeq 4.42 \mu_{B} / \mathrm{Mn}$, is obtained from the following expression:

$$
\mu_{\mathrm{eff}}=g \sqrt{x S_{1}\left(S_{1}+1\right)+(1-x) S_{2}\left(S_{2}+1\right)} .
$$

Here, $g=2$ is the Landé factor, $x$ is the $\mathrm{Sr}$ concentration, and $S_{1}=2$ and $S_{2}=3 / 2$ are the spin values of $\mathrm{Mn}^{4+}$ and $\mathrm{Mn}^{3+}$ ions, respectively. Therefore, one can conclude that the amorphous film is a typical paramagnet with free motion of the individual Mn spins. Such a magnetic behavior of the amorphous film is not unexpected. It is believed that the FM ground state in manganite is provided by Zener's doubleexchange interaction by means of an electron transfer from $\mathrm{Mn}^{3+}$ to $\mathrm{Mn}^{4+}$ the oxygen. The value of electronic transfer integral is determined mainly by the $\mathrm{Mn}-\mathrm{O}$ bond length and the $\mathrm{Mn}-\mathrm{O}-\mathrm{Mn}$ angle. The final result for magnetic exchange interaction can be approximately written in the following form: $k_{B} T_{C} \sim W \sim \cos (\varphi) / d_{\mathrm{Mn}-\mathrm{O}}^{3.5}$, where $k_{B}$ is the Boltzmann constant, $T_{C}$ is the Curie temperature, $W$ is the bandwidth, $\varphi$ is the tilt angle on the plane of bond, and $d_{\mathrm{Mn}-\mathrm{O}}$ is the $\mathrm{Mn}-\mathrm{O}$ bond length. ${ }^{20,21}$ Therefore, any perturbation in the translation symmetry of the crystal lattice of film can lead to the variation in $\varphi$ and $d_{\mathrm{Mn}-\mathrm{O}}$ and, consequently, results in the change in magnetic exchange interaction. Since the amorphous film has not a regular crystal lattice, $\cos (\varphi) \rightarrow 0$ and the long-range super- and double-exchange interactions between manganese moments are suppressed.

Figure 4 presents the $M(T)$ curves for the nanoclustered film measured at different applied magnetic fields. The magnetization value increases monotonically with decreasing temperature for all the applied fields, demonstrating the SPM behavior down to about $40 \mathrm{~K}$. Moreover, the ZFC and the FC $M(T)$ curves are not split in this temperature range. At the same time, a significant difference between ZFC and FC $M(T)$ curves occurs below $40 \mathrm{~K}$. A similar effect is frequently observed in an ensemble of SPM nanoparticles and can be explained in the framework of the Néel-Brown ${ }^{22,23}$ theory. According to this model, there is a critical temperature, called the blocking one, given by $T_{B}$ $=E_{A} /\left[\ln \left(\tau_{\mathrm{obs}} / \tau_{0}\right) k_{B}\right]$, above which the magnetic moments of the SPM particles move freely owing to thermal fluctuations while they are transformed into the blocked state at $T \leq T_{B}$. Here $E_{A}=K V$ is the energy barrier between two directions of 


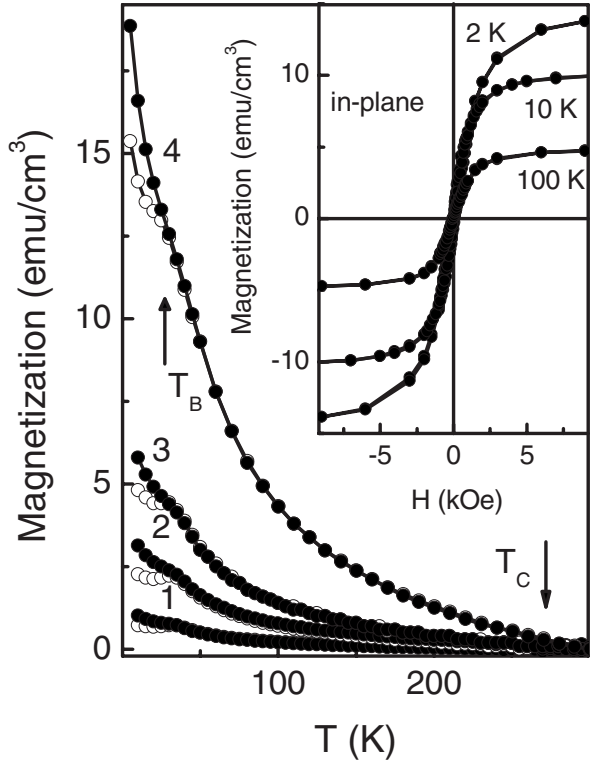

FIG. 4. Temperature dependence of the in-plane FC (solid symbols) and ZFC (open symbols) magnetization for the nanoclustered NSMO film, measured in applied magnetic fields of 500 Oe (1), 1500 Oe (2), 3000 Oe (3), and $10000 \mathrm{Oe}(4)$. Lines are guides to the eyes. Arrow indicates the location of the blocking temperature. The inset shows the in-plane hysteresis loops for the same film taken at 2,10 , and $100 \mathrm{~K}$.

the magnetic moment in a single SPM particle along and against an applied magnetic field. $K$ is the magnetic anisotropy constant and $V$ is the volume of SPM particle. $\tau_{\text {obs }}$ $\simeq 100 \mathrm{~s}$ is the observation time, $\tau_{0}$ is the characteristic time constant, called the attempt frequency, which is in the order of $10^{-10} \mathrm{~s}$ for $\mathrm{FM}$ and ferrimagnetic materials, and $k_{B}$ is the Boltzmann constant. Using the aforementioned equation for the blocking temperature and the average diameter of the nanocluster, obtained from ED pattern, one can estimate the anisotropy constant for the nanoclustered film to be $K \simeq 8.4$ $\times 10^{4} \mathrm{~J} \mathrm{~m}^{-3}$. The obtained value is coincident, in the sense of order of magnitude, with that of the bulk manganites, ${ }^{24}$ and is typical for superparamagnets. The inset shows the in-plane magnetization loops for this film at different temperatures. The $M(H)$ dependence manifests a nonhysteretic behavior at $100 \mathrm{~K}$, and a minor hysteretic one at 10 and $2 \mathrm{~K}$ with a coercive fields of $H_{c} \simeq \pm 40$ and $\pm 60 \mathrm{Oe}$, respectively. The SPM state in nanoclustered film appears because the translation symmetry of crystal lattice is disrupted at the cluster boundary and the FM state can be realized only inside the clusters, while with a significant suppression of the exchange magnetic interaction between them. Therefore, the maximum size of the FM regions, which occur in the PM phase below $T_{C}$, is limited by the average diameter of nanoclusters. Figure 5 shows in-plane FC (solid symbols) and ZFC (open symbols) temperature-dependent magnetization curves at different magnetic fields for the self-organized bilayer film, deposited at $750{ }^{\circ} \mathrm{C}$. The main feature of this film lies in the unique $M(T)$ behavior with an increase in the applied magnetic field. At a low field $(100 \mathrm{Oe})$ the $M(T)$ demonstrates a shape typical for the FM state (the magnetization tends to saturate at low temperatures), while at higher ones the SPM-like behavior is added (the magnetization keeps growing with decreasing temperature). Therefore, the

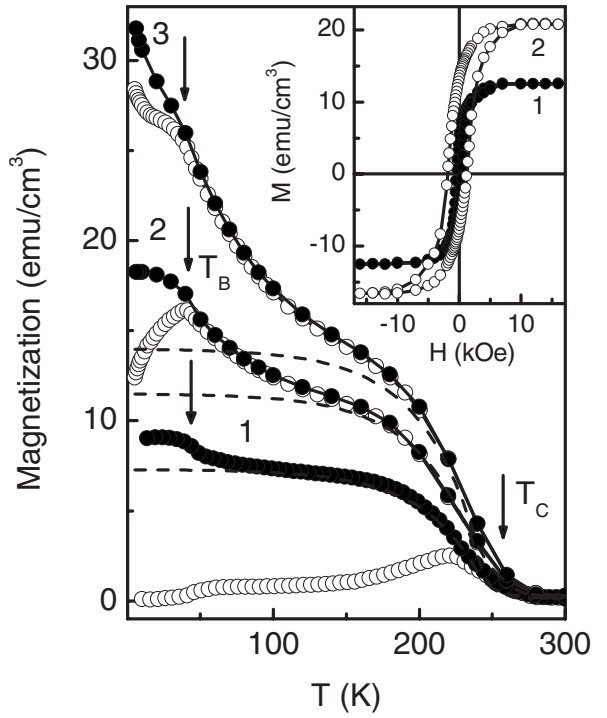

FIG. 5. Temperature dependence of the in-plane FC (solid symbols) and ZFC (open symbols) magnetization for the self-organized NSMO bilayer film, measured in an applied magnetic field of 100 Oe (1), 3000 Oe (2), and 10000 Oe (3). Lines are guides to the eyes. Arrows indicate the locations of the blocking $T_{B}$ and the Curie $T_{C}$ temperatures. The inset shows the in-plane hysteresis loops for the same film taken at $5 \mathrm{~K}$ (1) and $100 \mathrm{~K}$ (2). Lines are guides to the eyes.

$M(T)$ dependences testify as to the presence of two different magnetic phases, FM and SPM, which exist in different regions of the film. Taking into account the HREM analysis [Fig. 2(a)], one can conclude that the FM phase belongs to the bottom A layer, while the SPM one is relevant to the nanoclustered columnlike microstructure (B layer). Consequently, the total $M(T)$ dependence can be treated as a superposition of the FM and the SPM contributions. At low applied magnetic fields the $M(T)$ dependence manifests a splitting between FC and ZFC $M(T)$ curves right after the Curie point, $T_{C} \simeq 250 \mathrm{~K}$. Such a type of the $M(T)$ behavior is typical for the ferromagnets with a significantly large angle between the easy magnetization axis and the direction of applied magnetic field. It has been found that the easy axis magnetization for the manganite films is always along the direction of a tensile strain. ${ }^{25,26}$ According to the XRD and the HREM analyses, the bottom A layer in this film has the out-of-plane tensile strain. Hence, the easy axis magnetization is normal to the applied magnetic field, which is a main reason for the observed low-field ZFC and FC $M(T)$ irreversibility. When the applied magnetic field exceeds the coercive field $\left(H_{c}\right)$, this splitting disappears. At the same time, Fig. 5 shows a remarkable difference between ZFC and FC $M(T)$ below $T_{B} \simeq 50 \mathrm{~K}$ at all the applied magnetic fields. It is clear that this part of $M(T)$ dependence is related to the top B layer with a columnlike microstructure. In addition, it is necessary to draw attention to the lack of a sharp drop in the $M(T)$ near $T_{N} \simeq 150 \mathrm{~K}$, which is connected with the thermodynamical AFM transition. ${ }^{2,5}$ Therefore, it is suggested that the bottom A layer is the continuous FM one, while the top B one is SPM, which is formed by the FM clusters. It is coincident with the ED data, manifesting the absence of $\mathrm{CO}$ phase.

The inset in Fig. 5 displays the in-plane magnetization loops for this film, measured at different temperatures. The 


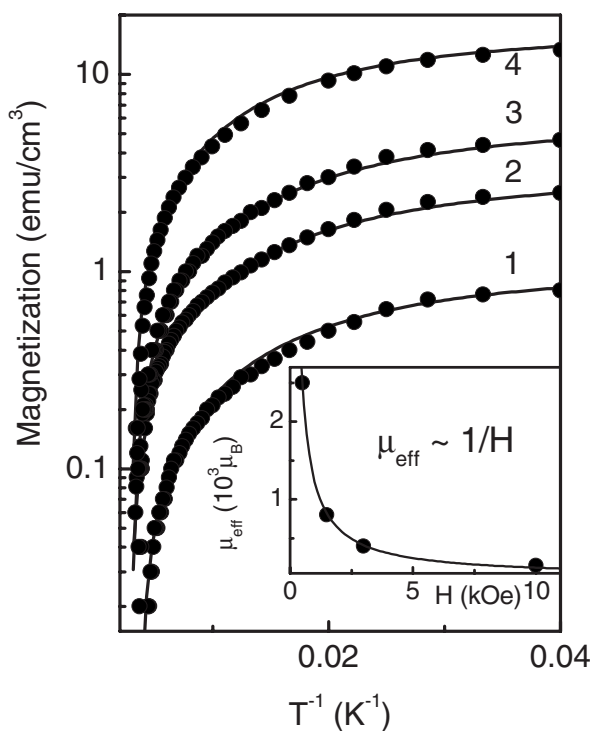

FIG. 6. $M(T)$ vs $1 / T$ plot for the nanoclustered NSMO film, taken at $H$ $=500$ Oe (1), 1500 Oe (2), 3000 Oe (3), and 10000 Oe (4). Solid lines represent the Langevin functions best fitted to the experiment data with a variation of the effective magnetic moment $\mu_{\text {eff. }}$ The inset displays the $\mu_{\mathrm{eff}}(H)$ dependence simulated on the basis of Langevin approximation.

$M(H)$ dependence demonstrates a hysteretic behavior at both temperatures, which is typical for the ferromagnet. At $100 \mathrm{~K}$ the magnetization loop has a symmetric shape with a coercive field of $H_{c} \simeq \pm 400 \mathrm{Oe}$, but becomes greatly asymmetric with decreasing temperature below $T_{B}: H_{C} \simeq+1200$ and $-1800 \mathrm{Oe}$ at $10 \mathrm{~K}$, for example. It is worth noting that the hysteresis loops were measured after cooling without an applied magnetic field.

\section{DISCUSSION}

Let us consider the peculiarity of the magnetic state in NSMO films, controlled by different kinds of microstructural disorder. Figure 3 reveals that the amorphous film is a typical paramagnet with free motion of the individual Mn spins, whose $M(T)$ dependence is well described with in the framework of CW approximation. Therefore, the quenched microstructural disorder, which is formed during the deposition at $T_{\text {sub }} \simeq 20{ }^{\circ} \mathrm{C}$, results in the PM state of film. Such effects have been observed previously in $\mathrm{La}_{0.7} \mathrm{Ca}_{0.3} \mathrm{MnO}_{3}$ films ${ }^{14}$ and can be explained by the anomalously small size of the crystallites and the full suppression of the long-range magnetic ordering.

In contrast to this ordering, for the nanoclustered film deposited at $T_{\text {sub }} \simeq 300{ }^{\circ} \mathrm{C}$, the temperature dependence of magnetization cannot be described by the $\mathrm{CW}$ approximation. Figure 6 shows the $M$ versus $1 / T$ plot for this film, measured at different applied magnetic fields. The plot does not demonstrate a linear behavior in the entire temperature range. Such nonlinear behavior of $M(H / T)$ is more typical for the SPM particles and is described, as a rule, by the Langevin function ${ }^{19}$

$$
M^{\mathrm{SPM}}(T, H)=M_{S}^{\mathrm{SPM}}\left[\operatorname{coth}\left(\frac{\mu_{\mathrm{eff}} H}{k_{B} T}\right)-\frac{k_{B} T}{\mu_{\mathrm{eff}} H}\right],
$$

where $M_{S}^{\mathrm{SPM}}$ is the saturation magnetization of the SPM phase and $\mu_{\text {eff }}$ is the average magnetic moment of the SPM particles. Solid lines in Fig. 6 represent the Langevin functions best fitted to the experimental data, with variation of the average effective magnetic moment $\mu_{\text {eff }}$. Surprisingly, $\mu_{\text {eff }}$ is strongly dependent on the applied magnetic field to be $\mu_{\text {eff }} \sim 1 / H$ empirically, as shown in the inset. By taking the saturation magnetic moment for the bulk NSMO in the FM state as $3.5 \mu_{B} / \mathrm{Mn}$ (Ref. 27) and assuming a spherical shape of the SPM clusters with a volume of $\pi D^{3} / 6$, their average diameters are estimated to be $D \simeq 4.3$ and $1.7 \mathrm{~nm}$ for magnetic fields of 0.05 and $1.0 \mathrm{~T}$, respectively. In principle, it is coincident with the ED data [see the inset in Fig. 1(b)], particularly in the case of the lowest applied magnetic field, and allows us to conclude that the nanoclustered disorder leads to formation of the SPM state in the film. However, the size of the SPM particle is not rigidly bound with the cluster and is controlled by an applied magnetic field, in contradiction to the underlying principles of the theoretical models for an ensemble of SPM particles. In addition, the fitting by the Langevin function of the $M(H)$ curves, shown in the inset of Fig. 4, reveals the unexpected strong temperature dependence of the effective magnetic moment, which decreases with decreasing temperature from $\mu_{\mathrm{eff}} \simeq 3000 \mu_{B}$ at $T$ $=100 \mathrm{~K}$ to $\mu_{\text {eff }} \simeq 40 \mu_{B}$ at $T=2 \mathrm{~K}$. It also contradicts the physics of magnetic materials where an increase in the magnetic moment with decreasing temperature occurs due to an enhancement of the FM exchange.

A similar problem in interpreting the experimental data in the framework of the classical Langevin model occurred in the case of high-density granular SPMs. ${ }^{28-31}$ It has been shown that the dipolar interaction plays a very important role for the magnetic properties for SPM ensembles with a sufficiently short distance between granules. Such a type of magnetic material was called the "interacting superparamagnets." The nanoclustered film can be treated as interacting SPM. It is believed that the FM ground state of the manganites is governed by Zener's double-exchange interaction by means of an electron transfer from $\mathrm{Mn}^{3+}$ to $\mathrm{Mn}^{4+}$ via oxygen. ${ }^{1}$ Since any structural defect, such as grain or twin boundary, for example, blocks up the electron motion, the FM coupling between the adjacent clusters will be also suppressed. Consequently, the individual crystallites in the nanoclustered film are separate magnetic domains with an abnormally short (a few lattice constants) interparticle distance. The classical dipolar energy can be described by

$$
E_{i j}=\frac{\vec{\mu}_{i} \cdot \vec{\mu}_{j}}{r_{i j}^{3}}-\frac{3\left(\vec{\mu}_{i} \cdot \vec{r}_{i j}\right)\left(\vec{\mu}_{i} \cdot \vec{r}_{i j}\right)}{r_{i j}^{5}},
$$

where $\vec{\mu}_{i}$ and $\vec{\mu}_{j}$ are the magnetic moments of different particles (clusters), and $r_{i j}$ is the interparticle distance. By assuming, for simplicity, that all the nanoclusters have equal magnetic moment and interparticle distance, and that the statistical average of $\left\langle\vec{\mu}_{i} \cdot \vec{\mu}_{j}\right\rangle$ along the field direction gives $\left\langle\vec{\mu}_{i} \cdot \vec{\mu}_{j}\right\rangle=\mu^{2}\left\langle\cos \left(\theta_{i j}\right)\right\rangle=\mu^{2}\left(M / M_{S}\right)^{2},{ }^{32}$ where $\mu$ is the magnetic moment of SPM particle and $\theta_{i j}$ is the angle between 
magnetic moments of adjacent clusters, the dipolar energy can be expressed to be $E_{D}=\mu^{2}\left(M / M_{S}\right)^{2} / r^{3}$. Let us estimate the maximum value of the dipolar energy for the nanoclustered film. In this case all the magnetic moments of the SPM particles should be directed parallel to an applied magnetic field and to each other. In other words, a magnetic system reaches the full saturation state, $M / M_{S}=1$. Once again, by taking the saturation magnetic moment for the bulk NSMO in the FM state as $3.5 \mu_{B} / \mathrm{Mn}$, considering a spherical shape of the SPM clusters with an average diameter $D \simeq 4 \mathrm{~nm}$ (according to the ED analysis) and using an interparticle distance $r \simeq 1.5 \mathrm{~nm}$, the maximum dipolar energy was estimated to be $E_{D} \simeq 1.3 \times 10^{-19} \mathrm{~J}$. This value is one order higher than that obtained for the granular SPMs,${ }^{29,31,33}$ which is explained by the abnormally small interparticle distance in our case. At the same time, the magnetic (Zeemann) energy, $E_{M}=\mu H$, which is included in the Langevin function [Eq. (3)], is $2.8 \times 10^{-20} \mathrm{~J}$ at an applied magnetic field of $1 \mathrm{~T}$. Therefore, the dipolar contribution, $E_{D}$, to the total energy is similar to or higher than the magnetic one, $E_{M}$, particularly at high magnetic fields. On the other hand, with decreasing the applied magnetic field $M / M_{S} \rightarrow 0$, leading to suppression of the interparticle dipolar interaction. This is coincident with the experimental results (see inset in Fig. 6), which reveal that $\mu_{\text {eff }}$ obtained by fitting the $M(T)$ curve at $H=500$ Oe $\left(\mu_{\text {eff }} \simeq 2500 \mu_{B}\right)$ is nearly coincident with the real value: $\mu$ $=3.5 \mu_{B}\left[\pi(D)^{3} / 6 V_{\text {cell }}\right] \simeq 2100 \mu_{B}$, where $V_{\text {cell }}$ is the unit cell volume.

Apart from the direct numerical simulations, the dipolar interaction is introduced by properly modifying the argument of Langevin function for the noninteracting SPM particles: $\mu_{\mathrm{eff}} H / k_{B} T$. It is believed that the dipolar interaction tends to keep a magnetic disorder in the SPM ensemble (similar to the thermal energy) and is opposed to the ordering effect by the external magnetic field. Consequently, it is more convenient to modify the argument in the Langevin function, in other words, to include the dipolar energy ${ }^{29,30}$

$$
\frac{\mu_{\mathrm{eff}} H}{k_{B} T} \rightarrow \frac{\mu H}{k_{B} T+E_{D}}=\frac{\mu H}{k_{B}\left(T+T_{D}\right)},
$$

where $T_{D}=\alpha \mu^{2}\left(M / M_{S}\right)^{2} / k_{B} r^{3}$ is the so-called dipolar temperature. $\alpha$ is a proportionality constant derived from the sum of all dipolar energy contributions. In this case, the following relation between the effective magnetic moment, produced by the classical Langevin function, and the real magnetic moment becomes $\mu_{\text {eff }}=\mu T /\left(T+T_{D}\right)$. According to this empirical expression, $\mu_{\mathrm{eff}}$ trends toward $\mu$ at sufficiently high temperatures, while quickly going down to zero with decreasing temperature, which is observed experimentally. Therefore, the nanoclustered film is the superparamagnet with a strong dipolar interaction.

Let us consider the magnetic properties of the selforganized bilayer film in more detail. Figure 5 shows that the temperature dependence of magnetization, $M(T)$, represents a superposition of two magnetic contributions-FM with a Curie point $T_{C} \simeq 250 \mathrm{~K}$, which belongs to the bottom A layer, and SPM with a blocking temperature $T_{B} \simeq 50 \mathrm{~K}$, which corresponds to the top B one. The FM contribution can be described in the framework of mean-field theory with

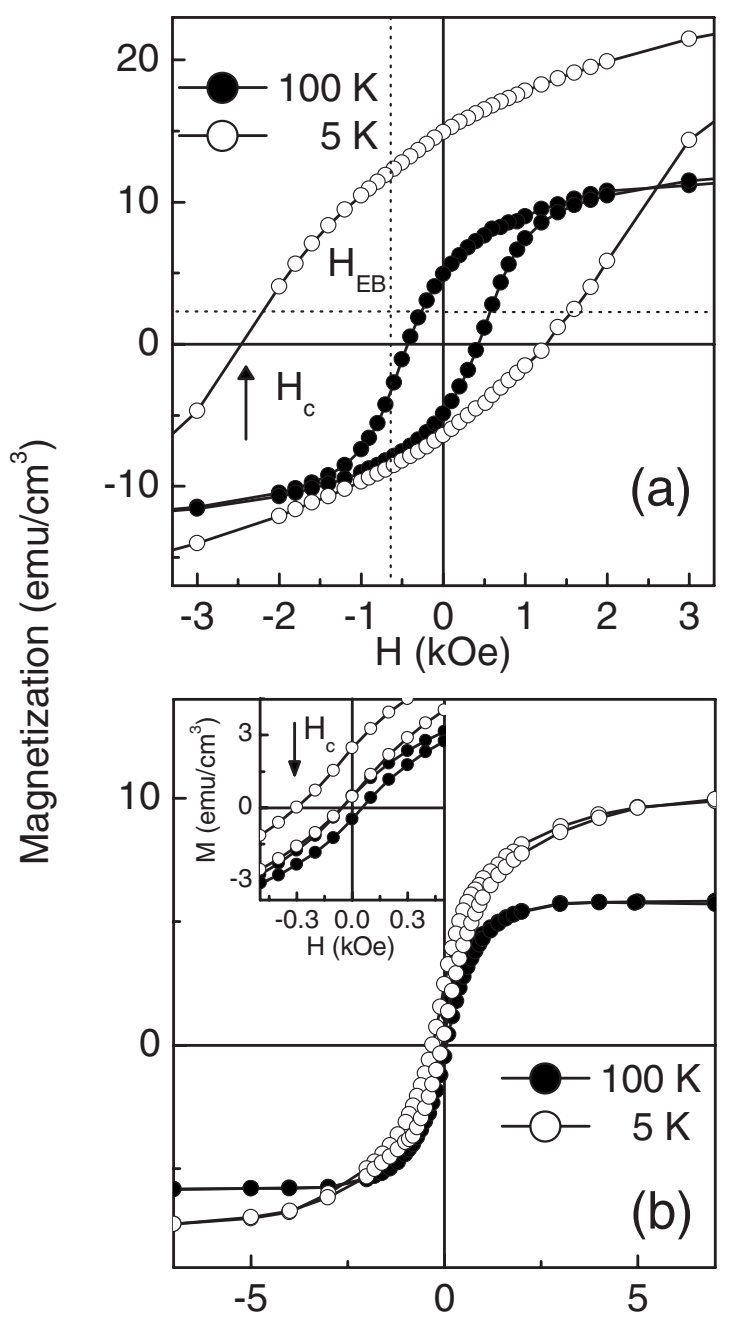

FIG. 7. (a) Low-field part of the in-plane hysteresis loops for the selforganized NSMO bilayer film taken at 5 and $100 \mathrm{~K}$. Arrow indicates the coercive field, $H_{c}$. Lines are guides to the eyes. Intersection of the dotted lines indicates the shift of hysteresis loop with respect to the symmetric form: $H_{\mathrm{EB}}$. (b) In-plane hysteresis loops for the self-organized NSMO bilayer film, deposited at $600{ }^{\circ} \mathrm{C}$. The inset displays the low-field part of the same hysteresis loops. Lines are guides to the eyes. Arrow indicates the coercive field $H_{c}$

only one fitting parameter of spontaneous magnetization at $T=0 .{ }^{19}$ The dashed lines in Fig. 5 are the theoretical $M^{\mathrm{FM}}(T)$ curves, which are best fitted with the experimental data, particularly near the Curie point. The fitting of the $M(T)$ dependences after the subtraction of the theoretical $M^{\mathrm{FM}}(T)$ curves with the Langevin function (not shown) reveals similar problems, which were raised in the analysis of the nanoclustered film: the effective magnetic moment $\mu_{\text {eff }}$ decreases with increasing magnetic field and decreasing temperature. The observed increase in $T_{B}$ in comparison with the nanoclustered film can be explained by a larger volume of the SPM clusters, which is confirmed by the HREM data [see Fig. 2(a)]. Therefore, we conclude that the interacting SPM state is realized in the top (B) layer of the self-organized bilayer film.

Inset in Fig. 5 shows that the in-plane hysteresis loop of the self-organized bilayer film becomes greatly asymmetric with decreasing temperature below $T_{B}$. Figure $7(\mathrm{a})$ displays the hysteresis loops obtained at 5 and $100 \mathrm{~K}$ in more detail. Moreover, the hysteresis loop undergoes a slight vertical 
shift along the magnetization axis. The saturation magnetization $\left(M_{S}\right)$ is symmetric to be $\pm 12.5 \mathrm{emu} / \mathrm{cm}^{3}$ at $100 \mathrm{~K}$ and asymmetric to be +21 and $-17 \mathrm{emu} / \mathrm{cm}^{3}$ at $5 \mathrm{~K}$. Taking into account that the hysteresis loops have been measured up to a magnetic field higher than the saturated one (at least, for the FM bottom layer), the observed phenomenon is not explained by the usual "minor loop" effect, which is provided by the incomplete transition to the saturated FM state and is typical for hard ferromagnets. ${ }^{34}$

More than fifty years ago, ${ }^{35}$ a shift of the hysteresis loop along the field axis was first observed for the FM/AFM magnetically coupled system and was called the "EB" interaction. It is generally accepted that the $\mathrm{EB}$, resulting from the exchange anisotropy at the FM/AFM interface, is provided by the coupling between the FM layer and the uncompensated interfacial spins in the AFM layer, the number of which determines the magnitude of exchange field $\left(H_{\mathrm{EB}}\right){ }^{36}$ The conventional EB is observed upon cooling the FM/AFM bilayer in the presence of a static magnetic field from a temperature above the Néel point $\left(T_{N}\right)$ but below the Curie one down to a certain temperature $T<T_{N}$. Since the selforganized NSMO bilayer film demonstrates a shift of the hysteresis loop, this (on formal signs) can be treated as an EB effect with $H_{\mathrm{EB}} \simeq-300$ and $-600 \mathrm{Oe}$ at 10 and $5 \mathrm{~K}$, respectively. However, in contrast to the traditional EB effect, both layers have a FM origin and the EB appears upon cooling without applied a magnetic field. The internal magnetic field originating from the magnetization state of the FM bottom layer plays the role of an applied field. ${ }^{37,38}$ It is necessary to note once more that both layers in the selforganized film have the same chemical composition and differ from each other only in the microstructure. Moreover, the A and the B layers keep the FM state down to low temperatures, which is confirmed by the following experimental evidence. First, the ED patterns, taken at $87 \mathrm{~K}$, do not manifest presence of the $\mathrm{CO}$ phase, which is accompanied by formation of the AFM state, as a rule. Second, the $M(T)$ magnetization curves, taken at different applied magnetic fields, do not demonstrate any peculiarity, relevant to the $\mathrm{FM} \rightarrow \mathrm{AFM}$ transition leading to a $\mathrm{ZFC} / \mathrm{FC}$ splitting at $T \leq T_{B}$, which is provided by the transition of the SPM $B$ layer into the blocking state. In addition, the hysteresis loop shift can be observed at a temperature below $T_{B}$, only when the blocking state is formed in the top layer. Consequently, the EB effect in our case is governed by the interaction between the blocked SPM and the FM layers over an interface, resulting in the shift of hysteresis loop along the magnetic field axis. To confirm that the shifted hysteresis loop corresponds only to the FM bottom $A$ layer, we prepared the same film at $T_{\text {sub }} \simeq 600{ }^{\circ} \mathrm{C}$. The microstructural analysis reveals (not shown) that the film has the same bilayer structure, containing the epitaxial (bottom) and the nanoclustered (top) layers. However, the thickness of bottom $A$ layer in this film does not exceed nanometers, which is quite smaller than that observed in the bilayer film, deposited at $T_{\text {sub }} \simeq 750{ }^{\circ} \mathrm{C}$. Figure 7(b) displays that even though the hysteresis loops of this film become narrower, the shift of $M(H)$ at $T \leq T_{B}$ can also be observed with $H_{\mathrm{EB}} \simeq-150$ Oe and $\Delta M_{S} \simeq+3 \mathrm{emu} / \mathrm{cm}^{3}$ at $5 \mathrm{~K}$. The detailed asymmetric shape of the hysteresis loop is represented in the inset. On the other hand, the inset in Fig. 4 shows that the nanoclustered film, without a uniform epitaxial FM $A$ layer, manifests only the symmetric $M(H)$ curves even at $T \leq T_{B}$. Therefore, the observed shift of the hysteresis loops is not an intrinsic property of the SPM layer.

The physical origin of the EB effect is based on the idea of a unidirectional anisotropy of the AFM spin plane, which is in contact with an FM layer over the FM/AFM interface. During the field cooling to $T<T_{N}$ all spins of this AFM plane are aligned ferromagnetically to those of the FM layer (assuming the FM exchange at the interface). Therefore, the internal magnetic field of the AFM spin plane comes to be in addition to or a subtraction from the external magnetic field at the magnetization reversal due to a sufficiently large AFM anisotropy, and provides the hysteresis loop shift. ${ }^{34}$ It is reasonable to suggest that in our case, the top $B$ layer in the SPM blocking state plays the role of AFM. At $T_{B}<T<T_{C}$ the magnetic moments of the SPM particles in the top B layer are randomly oriented owing to the thermal fluctuations, except at a thin area near the interface, which is influenced by the internal magnetic field of the FM bottom A layer. Consequently, due to the FM coupling between SPM and FM layers, a part of the SPM clusters is aligned ferromagnetically to those of the FM layer, and this state becomes frozen (or blocked) with decreasing temperature, $T<T_{B}$. Since we deal with the interacting SPM state, due to the strong dipolar interaction, these SPM clusters can be treated as a hard FM that pins the softer FM (bottom layer) via the exchange coupling at the magnetization reversal. ${ }^{39,40}$ Therefore, the observed hysteresis loop shift in the self-organized bilayer films is not a classical AFM/FM EB effect and is governed by a magnetic coupling between uniform FM and blocked interacting SPM layers.

\section{CONCLUSIONS}

The amorphous, nanoclustered, and self-organized bilayer NSMO films were prepared by rf magnetron sputtering using the so-called soft (or powder) target. The amorphous film was revealed as a typical paramagnet with freely moving individual $\mathrm{Mn}$ spins, the magnetic properties of which can be well described in the framework of CW approximation. The nanoclustered film manifests the magnetic properties that mimic an ensemble of SPM particles, but which cannot be described correctly in the framework of the classical Langevin approach. The observed contradictions in the magnetic behavior of the nanoclustered film was explained by a strong dipolar interaction between the SPM clusters. The self-organized bilayer films with a homogeneous chemical composition and different microstructures demonstrate the magnetic properties typical for the interacting SPM nanoparticles grown onto a continuous FM layer. The observed shift of the hysteresis loop at $T \leq T_{B}$ is evidence for the EB effect, which is explained by the different FM hardnesses of both layers. 


\section{ACKNOWLEDGMENTS}

This work was supported by the MEST/KOSEF through the Quantum Photonic Science Research Center, Korea. V. Svetchnikov is grateful to the financial support of Netherlands Institute for Metal Research.

${ }^{1}$ For the review, see, Colossal Magnetoresistance, Charge Ordering and Related Properties of Manganese Oxides, edited by C. N. R. Rao and B. Raveau (World Scientific, Singapore, 1998); Colossal Magnetoresistance Oxides, edited by Y. Tokura (Gordon and Breach, London, 1999); E. Dagotto, T. Hotta, and A. Moreo, Phys. Rep. 344, 1 (2001).

${ }^{2}$ R. Kajimoto, H. Yoshizawa, H. Kawano, H. Kuwahara, Y. Tokura, K. Ohoyama, and M. Ohashi, Phys. Rev. B 60, 9506 (1999).

${ }^{3}$ J. B. Goodenough, Structure and Bonding (Springer, Berlin/Heidelberg, 2001), Vol. 98.

${ }^{4}$ T. Fujiwara and M. Korotin, Phys. Rev. B 59, 9903 (1999).

${ }^{5}$ Y. Ogimoto, M. Nakamura, N. Takubo, H. Tamaru, M. Izumi, and K. Miyano, Phys. Rev. B 71, 060403 (2005).

${ }^{6}$ H. Wang, X. Zhang, M. F. Hudley, J. D. Thomoson, B. J. Gibbons, Y. Lin, P. N. Arendt, S. R. Foltyn, Q. X. Jia, and J. L. MacManus-Driscoll, Appl Phys. Lett. 84, 1147 (2004).

${ }^{7}$ Q. Qian, T. A. Tyson, C.-C. Kao, W. Prellier, J. Bai, A. Biswas, and R. L. Greene, Phys. Rev. B 63, 224424 (2001).

${ }^{8}$ C. Cui, T. A. Tyson, Z. Chen, and Z. Zhong, Phys. Rev. B 68, 214417 (2003).

${ }^{9}$ R. C. Yu, J. Tang, L. D. Yao, A. Matsushita, Y. Yu, F. Y. Li, and C. Q. Jin, J. Appl. Phys. 97, 083910 (2005).

${ }^{10}$ P. Levy, F. Parisi, G. Polla, D. Vega, G. Leyva, H. Lanza, R. S. Freitas, and L. Ghivelder, Phys. Rev. B 62, 6437 (2000).

${ }^{11}$ S. Dong, F. Gao, Z. Q. Wang, J. M. Liu, and Z. F. Ren, Appl. Phys. Lett. 90, 082508 (2007)

${ }^{12}$ V. Ponnambalam, S. Parashar, A. R. Raju, and C. N. R. Rao, Appl. Phys. Lett. 74, 206 (1999).

${ }^{13}$ V. G. Prokhorov, G. G. Kaminsky, V. A. Komashko, J. S. Park, and Y. P. Lee, J. Appl. Phys. 90, 1055 (2001).

${ }^{14}$ V. G. Prokhorov, V. A. Komashko, G. G. Kaminsky, V. L. Svetchnikov, Y. P. Lee, and S. Y. Park, Low Temp. Phys. 30, 938 (2004).

${ }^{15}$ W. Prellier, A. Biswas, M. Rajeswari, T. Venkatesan, and R. L. Greene, Appl. Phys. Lett. 75, 397 (1999).

${ }^{16}$ P. M. Woodward, D. E. Cox, T. Vogt, C. N. R. Rao, and A. K. Cheetham, Chem. Mater. 11, 3528 (1999).
${ }^{17}$ Y. P. Lee, S. Y. Park, Y. H. Hyun, J. B. Kim, V. G. Prokhorov, V. A. Komashko, and V. G. Svetchnikov, Phys. Rev. B 73, 224413 (2006).

${ }^{18}$ V. G. Prokhorov, V. A. Komashko, G. G. Kaminsky, Y. P. Lee, S. Y. Park, Y. H. Hyun, V. L. Svetchnikov, K. W. Kim, and J. Y. Rhee, Low Temp. Phys. 32, 128 (2006).

${ }^{19} \mathrm{C}$. L. Cullity, Introduction to Magnetic Materials (Addison-Wesley, New York, 1972)

${ }^{20}$ C. M. Varma, Phys. Rev. B 54, 7328 (1996).

${ }^{21}$ M. Medarde, M. Mesot, P. Lacorre, S. Rosenkranz, P. Fisher, and K. Grobcht, Phys. Rev. B 52, 9248 (1995).

${ }^{22}$ L. Néel, C. R. Acad. Sci. URSS 228, 664 (1949).

${ }^{23}$ W. F. Brown, Phys. Rev. 130, 1677 (1963).

${ }^{24}$ J. Mira, J. Rivas, M. Vázquez, M. R. Ibarra, R. Caciuffo, and M. A. S. Rodríguez, Europhys. Lett. 62, 433 (2003).

${ }^{25}$ T. K. Nath, R. A. Rao, D. Lavric, C. B. Eom, L. Wu, and F. Tsui, Appl. Phys. Lett. 74, 1615 (1999).

${ }^{26}$ R. Desfeux, S. Bailleul, A. Da Costa, W. Prellier, and M. Haghiri-Gosnet, Appl. Phys. Lett. 78, 3681 (2001).

${ }^{27}$ J. Geck, D. Buns, C. Hess, R. Klingeler, P. Reutler, M. v. Zimmermann, S.-W. Cheong, and B. Büchner, Phys. Rev. B 66, 184407 (2002).

${ }^{28}$ J. Du, B. Zhang, R. K. Zheng, and X. X. Zhang, Phys. Rev. B 75, 014415 (2007).

${ }^{29}$ P. Allia, M. Coisson, P. Tiberto, F. Vinai, M. Knobel, M. A. Novak, and W. C. Nunes, Phys. Rev. B 64, 144420 (2001).

${ }^{30}$ P. Allia, M. Coisson, J. Moya, P. Tiberto, and F. Vinai, J. Magn. Magn. Mater. 254-255, 143 (2003)

${ }^{31}$ O. Margeat, M. Tran, M. Spasova, and M. Farle, Phys. Rev. B 75, 134410 (2007).

${ }^{32}$ J. I. Gittleman, Y. Goldstein, and S. Bozowski, Phys. Rev. B 5, 3609 (1972).

${ }^{33}$ D. Kechrakos and K. N. Trohidou, Phys. Rev. B 62, 3941 (2000).

${ }^{34}$ J. Nogués, J. Sort, V. Langlais, V. Skumryev, S. Suriñach, J. S. Muñoz, and M. D. Baró, Phys. Rep. 422, 65 (2005).

${ }^{35}$ W. H. Meiklejohn and C. P. Bean, Phys. Rev. 102, 1413 (1956).

${ }^{36}$ A. E. Berkowitz and K. Takano, J. Magn. Magn. Mater. 200, 552 (1999).

${ }^{37}$ P. Miltényi, M. Gierlings, M. Bamming, U. May, G. Güntherodt, J. Nogués, M. Gruyters, C. Leighton, and I. K. Schuller, Appl. Phys. Lett. 75, 2304 (1999)

${ }^{38}$ S. Brück, J. Sort, V. Baltz, S. Suriñach, J. S. Muñoz, B. Dieny, M. D. Baró, and J. Nogués, Adv. Mater. (Weinheim, Ger.) 17, 2978 (2005).

${ }^{39}$ A. Berger, D. T. Margulies, and H. Do, Appl. Phys. Lett. 85, 1571 (2004).

${ }^{40} \mathrm{Ch}$. Binek, S. Polisetty, X. He, and A. Berger, Phys. Rev. Lett. 96, 067201 (2006). 